\title{
Tratamento Preservativo de Duas Espécies de Bambu por Imersão Prolongada e Boucherie Modificado
}

\author{
Rogy Frigeri Tiburtino ${ }^{1}$, Juarez Benigno Paes ${ }^{1}$, Antônio Ludovico Beraldo², \\ Marina Donaria Chaves Arantes ${ }^{1}$, Victor Fassina Brocco ${ }^{1}$ \\ ${ }^{1}$ Departamento de Ciências Florestais e da Madeira, Universidade Federal do Espírito Santo - UFES, \\ Jerônimo Monteiro/ES, Brasil
}

${ }^{2}$ Faculdade de Engenharia Agrícola, Universidade Estadual de Campinas - UNICAMP, Campinas/SP, Brasil

\section{RESUMO}

O objetivo desta pesquisa foi avaliar a qualidade do tratamento preservativo das espécies de bambu Dendrocalamus giganteus e Bambusa vulgaris submetidas a dois métodos de tratamento. Os bambus foram colhidos em touceiras em Alegre e Jerônimo Monteiro, sul do Espírito Santo. As hastes foram seccionadas em colmos de 2,0 m de comprimento e tratadas em soluções de $1 \mathrm{e}$ $3 \%$ de ingredientes ativos (i.a.) de um produto à base de cobre, cromo e boro (CCB). No método de imersão prolongada, os colmos foram imersos na solução por 5, 10 e 15 dias. No método de Boucherie modificado, a solução penetrou no bambu pela ação de uma pressão de 0,7 MPa. Foi avaliada a qualidade do tratamento por meio da penetração e da retenção do CCB nos colmos. Houve maior penetração de i.a. na base dos colmos e a retenção ficou abaixo da recomendada pela norma brasileira para tratamento de madeira.

Palavras-chave: qualidade do tratamento, Dendrocalamus giganteus, Bambusa vulgaris.

\section{Preservative Treatment of Two Bamboo Species by Long-Term Immersion and Modified Boucherie}

\begin{abstract}
This study aimed to evaluate the quality of preservative treatment of Bambusa vulgaris and Dendrocalamus giganteus bamboo species employing two treatment methods. The bamboos were collected in clumps in the municipalities of Alegre and Jerônimo Monteiro, southern of Espírito Santo State, Brazil. The stems were sectioned in $2.0 \mathrm{~m}$-long culms and treated in solutions with 1 and $3 \%$ of the active ingredients (a.i.) of a product based on copper, chromium and boron (CCB). In the long-term immersion method, the culms were immersed in the solution for 5, 10 and 15 days. In the modified Boucherie method, the solution penetrated the bamboo species using a pressure of $0.7 \mathrm{MPa}$. Treatment quality was evaluated by means of penetration and retention of CCB in the culms. There was greater penetration of a.i. at the base of the culms, and retention was below recommendation according to the Brazilian standards for wood treatment.
\end{abstract}

Keywords: treatment quality, Dendrocalamus giganteus, Bambusa vulgaris. 


\section{INTRODUÇÃO}

Em 2011, o governo brasileiro sancionou um projeto de lei que instituiu a Política Nacional de Incentivo ao Manejo Sustentado e ao Cultivo do Bambu, a "Lei do Bambu" n. ${ }^{0} 12.484$. Essa medida tem como objetivo estimular o manejo sustentado e o cultivo do bambu em pequenas e médias propriedades.

De acordo com Liese (1998), a estrutura anatômica do colmo do bambu determina suas propriedades, sendo o mesmo composto por nós e entrenós; os nós possuem células interligadas transversalmente, ocorrendo intensa ramificação dos vasos, e nos entrenós, não existem elementos celulares radiais, como raios, sendo as células axialmente orientadas.

O bambu, por possuir um elevado teor de amido na sua constituição, é susceptível ao ataque de xilófagos, sendo o coleóptero Dinoderus minutus aquele que frequentemente ataca o colmo da planta. Em função disto, após os devidos cuidados durante a colheita, os colmos devem ser submetidos a tratamentos preservativos. Assim, a durabilidade dos colmos pode ser influenciada pelo tratamento empregado (Azzini \& Beraldo, 2001). Os métodos de tratamento podem ocorrer de forma natural ou pelo emprego de produtos químicos.

Alguns tratamentos químicos podem ser efetuados utilizando um processo básico de substituição de seiva (capilaridade) ou de difusão, sendo os métodos de imersão prolongada e o de Boucherie modificado, exemplos destes processos. Para o tratamento químico, Santos \& Lopes (1998) e Azzini \& Beraldo (2001) recomendaram o uso de uma solução de $1 \%$ de concentração, preparada com sulfato de cobre, dicromato de sódio e ácido bórico.

Segundo Ubidia (2012), a imersão em água é um dos métodos de tratamento preservativos mais utilizados na América Latina. Quando o bambu é transportado por rios, o amido sofre lixiviação, diminuindo assim $\mathrm{o}$ ataque de insetos xilófagos.

O método de Boucherie modificado é outra forma popular de efetuar-se o tratamento preservativo do bambu. Nesse método, o preservativo é passado sob pressão por meio dos vasos, até que saia na outra extremidade do colmo. Essa prática deve ser aplicada apenas ao bambu recém-cortado, dentro de 24 horas após a colheita (Janssen, 2000).
Pereira \& Beraldo (2008) recomendaram alguns cuidados durante o tratamento com o método de Boucherie, pois a pressão aplicada em excesso pode causar a ruptura longitudinal do colmo em algumas espécies de bambu de paredes finas, e citam que uma pressão de $0,7 \mathrm{MPa}$ é satisfatória para a eficácia do tratamento.

A maior parte das pesquisas sobre o tratamento químico de bambu aplicando métodos tradicionais de preservação tem empregado produtos à base de cobre, cromo e boro (CCB), pois este é considerado menos nocivo à saúde do operador e ao ambiente, quando comparado com produtos que possuem arsênico em sua formulação.

O objetivo desta pesquisa foi avaliar a qualidade do tratamento preservativo das espécies de bambu Dendrocalamus giganteus e Bambusa vulgaris submetidas aos métodos de tratamento por imersão prolongada e Boucherie modificado.

\section{MATERIAL E MÉTODOS}

\subsection{Produto químico e métodos de tratamentos empregados}

Para o tratamento dos colmos, foi utilizado um produto hidrossolúvel, fungicida e inseticida à base de borato de cobre cromatado (CCB-Óxido), que possui boa mobilidade na madeira, sendo indicado para o tratamento de material verde. Foram utilizadas soluções de 1 e 3\% de ingredientes ativos do produto preservativo.

No método de imersão prolongada, os diafragmas dos colmos de bambu foram rompidos; esse procedimento foi realizado para auxiliar na absorção da solução preservativa e dificultar a flutuação dos mesmos na solução de tratamento. Os colmos foram expostos à solução preservativa por um período de 5, 10 e 15 dias.

Os colmos foram distribuídos em cochos $(2,10 \mathrm{~m}$ de comprimento $\times 0,44 \mathrm{~m}$ de largura $\times 0,56 \mathrm{~m}$ de profundidade), confeccionados com compensado resistente à umidade, tendo sido forrados internamente com lona plástica.

No método de Boucherie modificado, a solução preservativa penetrou no interior do lenho do bambu pela ação da força exercida pela pressão de ar de $0,7 \mathrm{MPa}$ liberada por um compressor. $\mathrm{O}$ ar comprimido liberado 
movimentou-se para o interior de uma autoclave piloto (19,5 cm de diâmetro e 53,0 cm de comprimento), na qual estava a solução química preservativa. A solução foi liberada por uma tubulação de PVC, que se conecta às válvulas de escape localizadas na autoclave e ao bambu, permitindo que o preservativo preenchesse a tubulação e o primeiro entrenó dos colmos.

Nesse método, não houve tratamentos divididos por tempos e o encerramento do tratamento ocorreu a partir do momento em que a solução preservativa houvesse atravessado todo o comprimento do colmo de bambu e cotejasse na extremidade oposta.

\subsection{Análises de penetração e retenção do $C C B$}

Foram retirados discos de $\pm 2,5 \mathrm{~cm}$ de espessura, em três posições, nos colmos $(0,50,1,00$ e 1,90 m da base). Nos discos retirados, foram realizadas análises químicas para a determinação da penetração dos elementos cobre e boro, e, em um disco adjacente ao obtido a $0,50 \mathrm{~m}$ da base dos colmos, foram retiradas amostras para a realização da análise de retenção dos i.a. do CCB, seguindo as recomendações da Norma Brasileira Regulamentadora - NBR 6232 da Associação Brasileira de Normas Técnicas - ABNT (2013).

Para a análise de penetração dos elementos cobre e boro, foi utilizado o método colorimétrico, em que foi aplicada, na superfície transversal de cada disco, uma solução de cromoazurol-S para detectar a presença do elemento cobre e, no lado oposto, foram empregadas soluções de álcool polivinílico e iodo para detectar o elemento boro.

A penetração dos elementos cobre e boro na peça tratada foi avaliada por meio de notas, sendo considerada a média da nota atribuída por cinco pessoas, de acordo com os padrões de penetração descritos por Sales-Campos et al. (2003), os quais sejam: penetração nula (nota $=0$ ); penetração vascular $(1)$; penetração parcial irregular (2); penetração parcial periférica (3), e penetração total (4) (Figura 1).

A determinação da quantidade de ingredientes ativos retidos nas amostras foi realizada seguindo a metodologia descrita por Wischer, citada por Paes et al. (2005) e Torres et al. (2011). Inicialmente, foram mensuradas a massa e o volume das amostras secas em estufa a $103 \pm 2^{\circ} \mathrm{C}$ por 48 horas; em seguida, essas amostras foram colocadas em mufla para serem incineradas à temperatura de $500-550^{\circ} \mathrm{C}$ por 4 horas, até
Penetração nula

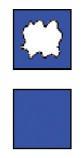

Penetração parcial periférica

Penetração vascular

Penetração total

Penetração parcial irregular

Figura 1. Padrões de penetração de preservativos na madeira (Sales-Campos et al., 2003).

Figure 1. Penetration patterns of preservatives in wood (Sales-Campos et al., 2003).

ocorrer a sua transformação em cinzas. Após remover o material da mufla, foram adicionados às cinzas $3 \mathrm{~mL}$ da mistura dos ácidos sulfúrico, perclórico e nítrico, todos concentrados, nas proporções 7:2:1.

Em uma chapa aquecida, foi realizada a digestão acelerada da mistura dos ácidos com as cinzas das amostras. $\mathrm{O}$ aquecimento ocorreu até que a mistura ficasse límpida, sendo diluídas as soluções ácidas obtidas em água destilada a volumes fixos de $100 \mathrm{~mL}$.

Após a diluição em água, os frascos foram identificados e enviados para realização das leituras, para quantificar os componentes do produto preservativo nas amostras. Na determinação do cobre e do cromo, foi empregado o espectrofotômetro de absorção atômica por emissão de chama; o boro foi analisado por fotocolorimetria. Para este trabalho, a retenção de $6,5 \mathrm{~kg}$ de i.a. $\mathrm{m}^{-3}$, recomendada pela NBR 9480 (ABNT, 2009) para o tratamento de madeiras de eucalipto para construções rurais, foi considerada como o valor mínimo.

Com os dados obtidos pela espectrofotometria e pela fotocolorimetria, e com o volume das amostras de bambu, os cálculos de retenção foram efetuados de acordo com a Equação 1, citada por Paes et al. (2005) e Torres et al. (2011).

$R=\frac{F x L \times F d \times 10^{-3}}{V}$

Em que:

$\mathrm{R}=$ Retenção do elemento no bambu $\left(\mathrm{kg}\right.$ i.a.m $\left.\mathrm{m}^{-3}\right)$;

$\mathrm{F}=$ Fator estequiométrico empregado para transformação dos elementos químicos para óxidos (cobre $\times 1,2518=\mathrm{CuO}$, cromo $\left.\times 1,9230=\mathrm{CrO}_{3}\right)$;

$\mathrm{L}=$ Leitura obtida do elemento químico $\left(\mathrm{mg} \cdot \mathrm{L}^{-1}\right)$;

$\mathrm{Fd}=$ Fator de diluição necessário para as leituras nos elementos;

$\mathrm{V}=$ Volume das amostras utilizadas nas análises $\left(\mathrm{cm}^{3}\right)$. 


\subsection{Análises estatísticas dos dados}

Para a penetração e a retenção do preservativo, foi empregado um delineamento inteiramente casualizado com arranjo fatorial, para a análise de variância entre os tratamentos. Na penetração do CCB, foram analisadas três posições nos colmos, empregando-se quatro repetições para cada método de tratamento.

Nas comparações estatísticas, os resultados de penetração da solução nas amostras tratadas pelo método de imersão prolongada foram analisados em função das posições nos colmos (três níveis), do tempo de tratamento (três níveis) e da concentração das soluções preservativas (dois níveis). No arranjo estatístico do método de Boucherie modificado, não foi empregado o tempo de tratamento. Para as análises estatísticas da retenção do CCB, somente as amostras coletadas a $0,50 \mathrm{~m}$ de altura da base de cada colmo foram utilizadas; assim, essa é a diferença para o arranjo da análise de penetração.

Para as análises, foram fixados os fatores método de tratamento e espécie, e para a penetração, também foi fixado o elemento químico (boro ou cobre). Os resultados de penetração foram transformados em raiz (notas $+0,5)$. Esta transformação foi necessária quando da necessidade de normalizar os dados e homogeneizar as variâncias. Foi empregado o teste de Tukey para comparação das médias, a 5\% de significância, para os fatores e as interações detectadas como sendo significativos pelo teste de $\mathrm{F}$.

\section{RESULTADOS E DISCUSSÃO}

\subsection{Penetração dos constituintes do CCB}

Observa-se, de maneira geral, para as espécies e as concentrações de ingredientes ativos avaliadas, que as notas mais elevadas para a penetração de cobre (Tabela 1) foram obtidas na posição da base dos colmos, seguida pelas posições do meio e do topo dos colmos. Este comportamento pode ser explicado, para o método de imersão prolongada, pela predisposição do sentido do fluxo ser ascendente no colmo, e para o de Boucherie modificado, além deste fator, pelo contato direto da base dos colmos com a solução preservativa.

O tratamento dos colmos de bambu com a concentração de $3 \%$ de ingredientes ativos obteve as maiores notas médias de penetrações do elemento cobre, nos dois métodos empregados. Fez-se exceção para o B. vulgaris, tratado pelo método de Boucherie

Tabela 1. Penetrações médias do elemento cobre por métodos de tratamento, espécies, concentrações da solução, tempos e posições nos colmos tratados.

Table 1. Penetration averages of the element copper by treatment methods, species, solution concentrations, times and positions in stems treated.

\begin{tabular}{|c|c|c|c|c|c|c|}
\hline \multirow{2}{*}{ Métodos } & \multirow{2}{*}{ Espécie } & \multirow{2}{*}{$\begin{array}{c}\text { Conc } \\
(\%)\end{array}$} & \multirow{2}{*}{$\begin{array}{l}\text { Tempo } \\
\text { (Dias) }\end{array}$} & \multicolumn{3}{|c|}{ Penetração (Nota) por Posição nos Colmos } \\
\hline & & & & Base & Meio & Topo \\
\hline \multirow{12}{*}{ Imersão Prolongada } & \multirow{6}{*}{$\begin{array}{c}\text { D. } \\
\text { giganteus }\end{array}$} & \multirow{3}{*}{1} & 5 & $1,00 \pm 0,16$ & $0,80 \pm 0,16$ & $0,25 \pm 0,25$ \\
\hline & & & 10 & $1,45 \pm 0,77$ & $1,00 \pm 0,67$ & $0,40 \pm 0,28$ \\
\hline & & & 15 & $1,25 \pm 0,19$ & $0,90 \pm 0,20$ & $0,20 \pm 0,16$ \\
\hline & & \multirow{3}{*}{3} & 5 & $1,95 \pm 1,36$ & $0,90 \pm 0,11$ & $0,35 \pm 0,34$ \\
\hline & & & 10 & $2,95 \pm 1,27$ & $1,05 \pm 0,19$ & $0,55 \pm 0,19$ \\
\hline & & & 15 & $2,30 \pm 0,38$ & $1,30 \pm 0,25$ & $0,95 \pm 0,41$ \\
\hline & \multirow{6}{*}{$\begin{array}{c}B . \\
\text { vulgaris }\end{array}$} & \multirow{3}{*}{1} & 5 & $1,50 \pm 0,52$ & $1,10 \pm 0,25$ & $1,00 \pm 0,32$ \\
\hline & & & 10 & $1,35 \pm 0,95$ & $0,70 \pm 0,38$ & $0,65 \pm 0,44$ \\
\hline & & & 15 & $1,45 \pm 0,64$ & $1,10 \pm 0,47$ & $0,95 \pm 0,55$ \\
\hline & & \multirow{3}{*}{3} & 5 & $1,60 \pm 0,51$ & $1,55 \pm 0,80$ & $1,00 \pm 0,71$ \\
\hline & & & 10 & $2,20 \pm 1,24$ & $1,35 \pm 0,41$ & $1,60 \pm 0,71$ \\
\hline & & & 15 & $2,50 \pm 1,06$ & $2,00 \pm 0,81$ & $1,05 \pm 0,41$ \\
\hline \multirow{4}{*}{ Boucherie Modificado } & \multirow{2}{*}{$\begin{array}{c}\text { D. } \\
\text { giganteus }\end{array}$} & 1 & --- & $0,25 \pm 0,30$ & $0,10 \pm 0,20$ & $0,10 \pm 0,20$ \\
\hline & & 3 & --- & $0,85 \pm 0,57$ & $0,75 \pm 0,50$ & $0,50 \pm 0,48$ \\
\hline & \multirow{2}{*}{$\begin{array}{c}B . \\
\text { vulgaris }\end{array}$} & 1 & --- & $0,65 \pm 0,44$ & $0,35 \pm 0,25$ & $0,45 \pm 0,34$ \\
\hline & & 3 & --- & $0,55 \pm 0,57$ & $0,35 \pm 0,44$ & $0,05 \pm 0,10$ \\
\hline
\end{tabular}

Conc $=$ Concentração da solução preservativa . 
modificado, em que as notas médias de penetração, na base e no meio dos colmos, foram maiores para a concentração de $1 \%$. Isto pode estar relacionado com a pressão de tratamento aplicada, que promoveu uma maior difusão das soluções menos concentradas nos colmos desta espécie.

Ferreira (2010) encontrou resultados semelhantes a essa pesquisa, ao avaliar a penetração do cobre nas regiões da base $(0,55 \mathrm{~m}$ de altura), meio $(1,10 \mathrm{~m}$ de altura) e topo (2,10 m de altura) em colmos de B. vulgaris tratados por um método de substituição de seiva por transpiração, durante 3, 6 e 9 dias.

Foi verificado, na Tabela 2, que, para os tratamentos de imersão prolongada e Boucherie modificado, as penetrações de boro (notas) foram semelhantes, nas três posições nos colmos, tendo sido maiores no B. vulgaris para ambos os métodos de tratamento empregados. Não foram encontradas referências quanto à penetração de boro em colmos de bambu para ambas as espécies estudadas.

Na Tabela 3, verifica-se que os fatores concentração, posição e as interações entre concentração e tempo, e tempo e posição foram significativos para penetração do elemento cobre nos colmos de D. giganteus. Para
B. vulgaris, os efeitos dos parâmetros concentração, tempo de tratamentos e a interação entre concentração e tempo foram significativos. Já para o elemento boro, não houve diferença para as espécies de bambu para nenhuma das situações analisadas.

As comparações entre médias de penetração para a espécie D. giganteus (Tabela 4) demonstraram que, para a concentração de $1 \%$ de i.a., não houve diferença entre os tempos de tratamento. No entanto, na concentração de $3 \%$, no tempo de 10 dias, foi observado melhor resultado de penetração do que aquele obtido para 15 dias. Este resultado pode estar relacionado com a variação de umidade, idade e posição da obtenção dos colmos na touceira de bambu, que são fontes de variabilidade entre os colmos tratados.

Para o efeito do tempo, notou-se que o tempo de 10 dias apresentou diferenças entre as concentrações, tendo os colmos tratados com 3\% de i.a. permitido uma maior penetração.

Ainda analisando a espécie D. giganteus (Tabela 4), o tempo de tratamento não mostrou diferença na penetração entre as três posições. Nos tempos de 5 e 15 dias, observou-se menor penetração no meio dos colmos. A maior penetração na base e no topo

Tabela 2. Penetrações médias do boro por métodos de tratamento, espécies, concentrações da solução, tempos e posições nos colmos tratados.

Table 2. Penetration averages of boron by treatment methods, species, solution concentrations, times and positions in stems treated.

\begin{tabular}{|c|c|c|c|c|c|c|}
\hline \multirow{2}{*}{ Métodos } & \multirow{2}{*}{ Espécie } & \multirow{2}{*}{$\begin{array}{c}\text { Conc } \\
(\%)\end{array}$} & \multirow{2}{*}{$\begin{array}{l}\text { Tempo } \\
\text { (Dias) }\end{array}$} & \multicolumn{3}{|c|}{ Penetração (Nota) por Posição nos Colmos } \\
\hline & & & & Base & Meio & Topo \\
\hline \multirow{12}{*}{ Imersão Prolongada } & \multirow{6}{*}{$\begin{array}{c}D . \\
\text { giganteus }\end{array}$} & \multirow{3}{*}{1} & 5 & $2,20 \pm 0,67$ & $2,15 \pm 0,57$ & $2,10 \pm 0,58$ \\
\hline & & & 10 & $2,55 \pm 0,79$ & $2,35 \pm 0,81$ & $2,15 \pm 0,66$ \\
\hline & & & 15 & $2,25 \pm 0,91$ & $2,30 \pm 0,76$ & $2,35 \pm 0,57$ \\
\hline & & \multirow{3}{*}{3} & 5 & $2,30 \pm 0,84$ & $2,00 \pm 0,54$ & $2,40 \pm 0,23$ \\
\hline & & & 10 & $2,30 \pm 0,48$ & $2,10 \pm 0,35$ & $2,75 \pm 0,25$ \\
\hline & & & 15 & $2,40 \pm 0,52$ & $2,40 \pm 0,43$ & $2,50 \pm 0,50$ \\
\hline & \multirow{6}{*}{$\begin{array}{c}\text { B. } \\
\text { vulgaris }\end{array}$} & \multirow{3}{*}{1} & 5 & $3,40 \pm 0,52$ & $3,20 \pm 0,49$ & $3,35 \pm 0,53$ \\
\hline & & & 10 & $3,35 \pm 0,34$ & $3,30 \pm 0,48$ & $3,70 \pm 0,26$ \\
\hline & & & 15 & $3,05 \pm 0,77$ & $3,10 \pm 0,89$ & $3,20 \pm 0,73$ \\
\hline & & \multirow{3}{*}{3} & 5 & $3,20 \pm 0,37$ & $2,90 \pm 0,53$ & $3,40 \pm 0,54$ \\
\hline & & & 10 & $3,85 \pm 0,30$ & $3,70 \pm 0,35$ & $3,85 \pm 0,30$ \\
\hline & & & 15 & $3,50 \pm 0,60$ & $3,45 \pm 0,72$ & $3,35 \pm 0,77$ \\
\hline \multirow{4}{*}{ Boucherie Modificado } & \multirow{2}{*}{$\begin{array}{c}D . \\
\text { giganteus }\end{array}$} & 1 & --- & $1,55 \pm 0,55$ & $1,55 \pm 0,34$ & $1,05 \pm 0,10$ \\
\hline & & 3 & --- & $2,95 \pm 0,25$ & $2,70 \pm 0,38$ & $1,80 \pm 0,63$ \\
\hline & \multirow{2}{*}{$\begin{array}{c}B . \\
\text { vulgaris }\end{array}$} & 1 & --- & $3,05 \pm 0,62$ & $2,90 \pm 0,48$ & $3,15 \pm 0,19$ \\
\hline & & 3 & --- & $3,30 \pm 0,42$ & $3,20 \pm 0,59$ & $2,75 \pm 0,34$ \\
\hline
\end{tabular}

Conc = Concentração da solução preservativa. 
Tabela 3. Resumo das análises de variância da penetração de cobre e boro (nota) para o método de imersão prolongada. Table 3. Summary of the analyzes of variance of penetration of copper and boron (note) to long-term immersion method.

\begin{tabular}{|c|c|c|c|c|c|}
\hline \multirow{4}{*}{$\begin{array}{l}\text { Fontes de } \\
\text { Variação }\end{array}$} & \multirow{4}{*}{ Graus de Liberdade } & \multicolumn{4}{|c|}{ Imersão Prolongada } \\
\hline & & \multicolumn{4}{|c|}{ Quadrados Médios } \\
\hline & & \multicolumn{2}{|c|}{ Cobre } & \multicolumn{2}{|c|}{ Boro } \\
\hline & & D. giganteus & B.vulgaris & D. giganteus & B.vulgaris \\
\hline Concentração (Conc) & 1 & $0,234^{* *}$ & $1,186^{\star *}$ & $0,016^{\mathrm{NS}}$ & $1,616^{\mathrm{NS}}$ \\
\hline Tempo & 2 & $0,021^{\mathrm{NS}}$ & $0,481^{\star *}$ & $0,022^{\mathrm{NS}}$ & $3,449^{\mathrm{NS}}$ \\
\hline Posição (Pos) & 2 & $0,192^{* *}$ & $0,022^{\mathrm{NS}}$ & $0,015^{\mathrm{NS}}$ & $0,764^{\mathrm{NS}}$ \\
\hline Conc ${ }^{\star}$ Tempo & 2 & $0,076^{*}$ & $0,310^{\star *}$ & $0,001^{\mathrm{NS}}$ & $1,454^{\mathrm{NS}}$ \\
\hline Conc ${ }^{\star}$ Pos & 2 & $0,039^{\mathrm{NS}}$ & $0,006^{\mathrm{NS}}$ & $0,030^{\mathrm{NS}}$ & $0,098^{\mathrm{NS}}$ \\
\hline Tempo*Pos & 4 & $0,017^{*}$ & $0,024^{\mathrm{NS}}$ & $0,003^{\mathrm{NS}}$ & $0,218^{\mathrm{NS}}$ \\
\hline Conc $^{\star}$ Tempo ${ }^{\star}$ Pos & 4 & $0,564^{\mathrm{NS}}$ & $0,017^{\mathrm{NS}}$ & $0,011^{\mathrm{NS}}$ & $0,235^{\mathrm{NS}}$ \\
\hline Resíduo & 54 & 0,022 & 0,041 & 0,034 & 0,022 \\
\hline
\end{tabular}

${ }^{* *}$ significativo a $1 \%$ de probabilidade; * significativo a $5 \%$ de probabilidade; ${ }^{N s}$ não significativo a $5 \%$ de probabilidade.

Tabela 4. Penetrações médias do cobre para concentração, tempo e posição no colmo nas espécies D. giganteus e B. vulgaris, no método de imersão prolongada.

Table 4. Penetrations average copper concentration, time and position in the stem species D. giganteus and B. vulgaris to long-term immersion method.

\begin{tabular}{|c|c|c|c|}
\hline \multirow{3}{*}{ Concentração (\%) } & \multicolumn{3}{|c|}{ Imersão Prolongada/D. giganteus } \\
\hline & \multicolumn{3}{|c|}{ Tempo (Dias)/Penetração (Nota) } \\
\hline & 5 & 10 & 15 \\
\hline 1 & $0,77 \mathrm{Aa}$ & $0,76 \mathrm{Ab}$ & $0,82 \mathrm{Aa}$ \\
\hline 3 & $0,87 \mathrm{ABa}$ & $1,00 \mathrm{Aa}$ & $0,82 \mathrm{Ba}$ \\
\hline \multirow{2}{*}{ Tempo (Dias) } & \multicolumn{3}{|c|}{ Posição/Penetração (Nota) } \\
\hline & Base & Meio & Topo \\
\hline 5 & $0,78 \mathrm{ABa}$ & $0,75 \mathrm{Ba}$ & $0,93 \mathrm{Aa}$ \\
\hline 10 & $0,86 \mathrm{Aa}$ & $0,80 \mathrm{Aa}$ & $0,96 \mathrm{Aa}$ \\
\hline 15 & $0,81 \mathrm{ABa}$ & $0,73 \mathrm{Ba}$ & $0,92 \mathrm{Aa}$ \\
\hline \multirow{3}{*}{ Concentração (\%) } & & vulgaris & \\
\hline & \multicolumn{3}{|c|}{ Tempo (Dias)/Penetração (Nota) } \\
\hline & 5 & 10 & 15 \\
\hline 1 & $1,10 \mathrm{Bb}$ & $1,25 \mathrm{Bb}$ & $1,47 \mathrm{Aa}$ \\
\hline 3 & $1,37 \mathrm{Ba}$ & $1,72 \mathrm{Aa}$ & $1,50 \mathrm{Ba}$ \\
\hline
\end{tabular}

As médias seguidas da mesma letra, maiúscula na horizontal ou minúscula na vertical, para cada parâmetro, não diferem estatisticamente pelo teste de Tukey ( $\mathrm{p} \geq 0,05)$.

é causada pelo contato direto destas posições com a solução preservativa.

Já o tempo de 15 dias, com 1\% de i.a., na espécie B. vulgaris, proporcionou maior penetração, enquanto na concentração de $3 \%$ de i.a., o tempo de 10 dias obteve uma melhor penetração. E, nos tempos de 5 e 10 dias, os valores da concentração de 3\% i.a. foram superiores aos de $1 \%$.

Observa-se, na Tabela 5, que a penetração de cobre e boro, nos colmos submetidos ao método de Boucherie modificado, proporcionou efeitos significativos apenas para a espécie D. giganteus. Para esta espécie, os parâmetros concentração (cobre e boro) e posição proporcionaram efeitos significativos na penetração nos colmos.

Observa-se, na Tabela 6, que, na concentração de $3 \%$ de i.a., obteve-se maior penetração para cada elemento químico e as posições da base e do meio exibiram maiores penetrações do boro.

Uma explicação para a baixa penetração dos elementos do CCB nos colmos de bambu, principalmente o cobre, utilizando o Boucherie modificado como 
Tabela 5. Resumo das análises de variância da penetração de cobre e boro (nota) para o método de Boucherie modificado. Table 5. Summary of the analyzes of variance of penetration of copper and boron (note) to Boucherie modified method.

\begin{tabular}{|c|c|c|c|c|c|}
\hline \multirow{4}{*}{$\begin{array}{l}\text { Fontes de } \\
\text { Variação }\end{array}$} & \multirow{4}{*}{$\begin{array}{c}\text { Graus de } \\
\text { Liberdade }\end{array}$} & \multicolumn{4}{|c|}{ Boucherie Modificado } \\
\hline & & \multicolumn{4}{|c|}{ Quadrados Médios } \\
\hline & & \multicolumn{2}{|c|}{ Cobre } & \multicolumn{2}{|c|}{ Boro } \\
\hline & & D. giganteus & B. vulgaris & D. giganteus & B. vulgaris \\
\hline Concentração (Conc) & 1 & $0,448^{\star *}$ & $0,055^{\mathrm{NS}}$ & $0,741^{\star \star}$ & $0,001^{\mathrm{NS}}$ \\
\hline Posição & 2 & $0,030^{\mathrm{NS}}$ & $0,065^{\mathrm{NS}}$ & $0,166^{\star *}$ & $0,007^{\mathrm{NS}}$ \\
\hline Conc ${ }^{\star}$ Posição & 2 & $0,007^{\mathrm{NS}}$ & $0,025^{\mathrm{NS}}$ & $0,015^{\mathrm{NS}}$ & $0,022^{\mathrm{NS}}$ \\
\hline Resíduo & 18 & 0,045 & 0,039 & 0,019 & 0,016 \\
\hline
\end{tabular}

${ }^{* *}$ significativo a $1 \%$ de probabilidade; ${ }^{*}$ significativo a $5 \%$ de probabilidade; ${ }^{\text {NS }}$ não significativo a $5 \%$ de probabilidade.

Tabela 6. Penetrações médias do cobre e boro para concentração e posição no colmo da espécie D. giganteus, no método de Boucherie modificado.

Table 6. Penetration averages for copper and boron concentration and position in the stem of the species D. giganteus to modified Boucherie method.

\begin{tabular}{|ccc|}
\multirow{2}{*}{$\begin{array}{c}\text { Concentração } \\
(\%)\end{array}$} & \multicolumn{2}{c|}{ Boucherie Modificado } \\
\cline { 2 - 3 } & \multicolumn{2}{c|}{ Penetração (Nota) } \\
\cline { 2 - 3 } 1 & Cobre & Boro \\
\hline 3 & $0,80 \mathrm{~b}$ & $1,37 \mathrm{~b}$ \\
\hline Posição & $1,07 \mathrm{a}$ & $1,71 \mathrm{a}$ \\
\hline Base & \multicolumn{2}{c|}{ Penetração/boro (Nota) } \\
\hline Meio & \multicolumn{2}{c}{$1,64 \mathrm{a}$} \\
\hline Topo & \multicolumn{2}{c|}{$1,60 \mathrm{a}$} \\
\hline
\end{tabular}

As médias seguidas da mesma letra, para cada parâmetro, não diferem estatisticamente pelo teste de Tukey ( $\mathrm{p} \geq 0,05)$.

método de tratamento, pode estar associada ao tempo de tratamento. Neste trabalho, o período de tratamento variou de 15 a 25 minutos. $\mathrm{O}$ produto químico à base de CCB necessita de um tempo maior do que o descrito acima para se fixar no interior do bambu. Não constam informações na literatura sobre trabalhos que tenham avaliado o tempo de tratamento de bambu pelo método de Boucherie empregado nesta pesquisa.

\subsection{Retenção dos constituintes do $C C B$}

Nota-se, na Tabela 7, que as médias de retenção da concentração de $3 \%$ de i.a. foram superiores nos dois tratamentos testados, exceto para o D. giganteus, tratado durante 10 dias no método de imersão prolongada. Isto pode estar associado à variabilidade existente entre os colmos tratados.
As médias de retenção observadas no método de Boucherie modificado foram as menores. Esses resultados podem ser consequência de limitada duração do tratamento, conforme já discutido anteriormente.

Observa-se, de maneira geral, que as médias de retenção da espécie $B$. vulgaris foram mais elevadas do que as da espécie D. giganteus, nos tratamentos estudados. Levando-se em consideração os resultados de retenção, pode-se afirmar que, de maneira geral, a quantidade de ingredientes ativos retida nos bambus mostra-se inferior a $6,5 \mathrm{~kg}$ de i.a.m ${ }^{-3}$, valor mínimo recomendado pela NBR 9480 (ABNT, 2009) para o tratamento de madeiras de eucalipto para construções rurais.

A estrutura anatômica do bambu pode explicar os baixos valores de retenção observados neste estudo. $\mathrm{O}$ bambu, ao contrário da madeira, não possui elementos anatômicos dispostos na direção radial, sendo os vasos o principal meio de movimentação dos líquidos no interior dos colmos. Segundo Liese (1985), os vasos ocupam apenas $10 \%$ da área lenhosa do bambu. Espelho (2007) relacionou alguns fatores, tais como: a posição na touceira, quanto o nível de exposição à luz solar, a época de emergência do broto do solo e o aparecimento de "madeira de reação", que poderiam afetar as dimensões dos elementos anatômicos do colmo, influenciando, dessa forma, a eficiência do tratamento químico.

Nota-se, na Tabela 8, que a interação entre a concentração e o tempo de tratamento foi significativa para o D. giganteus, enquanto para o B. vulgaris, todos os efeitos analisados foram significativos. As interações foram desdobradas e as médias dos fatores, comparadas (Tabela 9). 
Tabela 7. Retenção média do preservativo CCB por métodos de tratamentos, espécies, tempos e concentração das soluções testadas.

Table 7. Retention averages of CCB by treatment methods, species, times solution concentrations of tested solutions.

\begin{tabular}{|c|c|c|c|c|}
\hline \multirow{3}{*}{ Métodos } & \multirow{3}{*}{ Espécie } & \multirow{3}{*}{$\begin{array}{c}\text { Tempo Tratamento } \\
\text { (Dias) }\end{array}$} & \multicolumn{2}{|c|}{ Retenção Média de CCB (kg i.a.m³) } \\
\hline & & & \multicolumn{2}{|c|}{ Concentração da Solução (\%) } \\
\hline & & & 1 & 3 \\
\hline \multirow{6}{*}{ Imersão Prolongada } & \multirow{3}{*}{ D. giganteus } & 5 & $0,60 \pm 0,34$ & $1,48 \pm 0,74$ \\
\hline & & 10 & $1,43 \pm 0,03$ & $0,63 \pm 0,08$ \\
\hline & & 15 & $0,45 \pm 0,30$ & $1,72 \pm 0,15$ \\
\hline & \multirow{3}{*}{ B. vulgaris } & 5 & $0,68 \pm 0,61$ & $1,93 \pm 0,32$ \\
\hline & & 10 & $0,98 \pm 0,35$ & $4,26 \pm 1,60$ \\
\hline & & 15 & $2,20 \pm 1,01$ & $10,80 \pm 0,67$ \\
\hline \multirow{2}{*}{ Boucherie Modificado } & D. giganteus & --- & $0,28 \pm 0,02$ & $1,40 \pm 0,26$ \\
\hline & B. vulgaris & --- & $0,84 \pm 0,18$ & $2,14 \pm 1,72$ \\
\hline
\end{tabular}

Tabela 8. Resumo das análises de variância da retenção $\left(\mathrm{kg}\right.$ i.a. $\left.\mathrm{m}^{-3}\right)$ para o preservativo à base de $\mathrm{CCB}$, para o método de imersão prolongada.

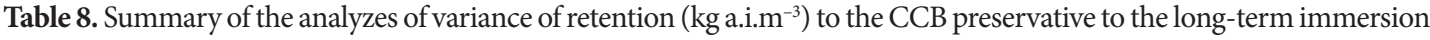
method.

\begin{tabular}{|c|c|c|c|}
\hline \multirow{3}{*}{$\begin{array}{l}\text { Fontes de } \\
\text { Variação }\end{array}$} & \multirow{3}{*}{$\begin{array}{c}\text { Graus de } \\
\text { Liberdade }\end{array}$} & \multicolumn{2}{|c|}{ Quadrado Médio } \\
\hline & & \multicolumn{2}{|c|}{ Imersão Prolongada } \\
\hline & & D. giganteus & B. vulgaris \\
\hline Concentração (Conc) & 1 & $0,602^{\mathrm{NS}}$ & $57,517^{\star *}$ \\
\hline Tempo & 2 & $0,003^{\mathrm{NS}}$ & $29,145^{\star *}$ \\
\hline Conc ${ }^{\star}$ Tempo & 2 & $1,218^{*}$ & $14,454^{\star *}$ \\
\hline Resíduo & 6 & 0,133 & 0,769 \\
\hline
\end{tabular}

** significativo a $1 \%$ de probabilidade; ${ }^{*}$ significativo a $5 \%$ de probabilidade; ${ }^{\text {Ns }}$ não significativo a $5 \%$ de probabilidade.

Tabela 9. Retenções médias para concentração e tempo de tratamento nos colmos, para as espécies estudadas submetidas ao método de imersão prolongada.

Table 9. Retentions averages for concentration and treatment time on the stems for the studied species submitted to long-term immersion method.

\begin{tabular}{|c|c|c|c|}
\hline \multirow{4}{*}{ Concentração (\%) } & \multicolumn{3}{|c|}{ Imersão Prolongada } \\
\hline & \multicolumn{3}{|c|}{ Dendrocalamus giganteus } \\
\hline & \multicolumn{3}{|c|}{ Tempo (Dias)/Retenções (kg i.a.m ${ }^{-3}$ ) } \\
\hline & 5 & 10 & 15 \\
\hline 1 & $0,61 \mathrm{Aa}$ & $0,63 \mathrm{Aa}$ & $0,45 \mathrm{Ab}$ \\
\hline 3 & $1,48 \mathrm{Aa}$ & $1,43 \mathrm{Aa}$ & $1,72 \mathrm{Aa}$ \\
\hline \multirow{3}{*}{ Concentração (\%) } & \multicolumn{3}{|c|}{ Bambusa vulgaris } \\
\hline & \multicolumn{3}{|c|}{ Tempo (Dias)/Retenções (kg i.a.m ${ }^{-3}$ ) } \\
\hline & 5 & 10 & 15 \\
\hline 1 & $0,68 \mathrm{Aa}$ & $0,98 \mathrm{Ab}$ & $2,19 \mathrm{Ab}$ \\
\hline 3 & $1,93 \mathrm{Ba}$ & $4,27 \mathrm{Ba}$ & $10,80 \mathrm{Aa}$ \\
\hline
\end{tabular}

As médias seguidas da mesma letra, maiúscula na horizontal ou minúscula na vertical, não diferem estatisticamente pelo teste de Tukey $(\mathrm{p} \geq 0,05)$.

Em ambas as espécies, a concentração de 3\% de i.a. exibiu maior retenção do preservativo (Tabela 9). No D. giganteus, a única diferença estatística que ocorreu entre as concentrações foi no tempo de 15 dias; já no B. vulgaris, foi observada diferença significativa para os tempos de 10 e 15 dias, ambas maiores na concentração de $3 \%$ i.a.

A espécie de bambu Bambusa blumeana foi tratada com ácido bórico em quatro concentrações, por imersão prolongada; na análise de retenção, foram encontradas 
quantidades de 2,15; 3,58; 5,19 e 11,03 kg.m $\mathrm{m}^{-3}$, utilizandose 1, 2, 4 e $8 \%$ de ácido bórico, respectivamente (Adrianus et al., 2010). Rosa et al. (2009) obtiveram uma retenção de $9,04 \mathrm{~kg} \cdot \mathrm{m}^{-3}$ no B. vulgaris, em concentração de 3\% de i.a. de CCB, realizando o tratamento pelo método de difusão, em que os colmos foram imersos em solução por $2 \mathrm{~h}$ e, em seguida, envolvidos por lonas plásticas durante quatro semanas.

O resumo das análises de variância para os colmos submetidos ao método de Boucherie (Tabela 10) indicou que a concentração da solução causou efeito significativo apenas para a espécie D. giganteus. Observa-se (Tabela 11) que os colmos tratados em solução com concentração de 3\% de i.a. tiveram uma retenção média mais elevada na espécie D. giganteus.

Como foi observado anteriormente, houve pouca penetração dos elementos cobre e boro, e os baixos valores de retenção nos colmos tratados por Boucherie comprovam a pouca eficiência desse método de tratamento, necessitando-se que as concentrações de ingredientes ativos e o tempo de tratamentos sejam mais elevados, a fim de se obterem retenções satisfatórias.

Tabela 10. Resumo das análises de variância da retenção $\left(\mathrm{kg}\right.$ i.a. $\left.\mathrm{m}^{-3}\right)$ para o preservativo à base de $\mathrm{CCB}$, para o método de Boucherie modificado.

Table 10. Summary of the analyzes of variance of retention $\left(\mathrm{kg}\right.$ i.a. $\left.\mathrm{m}^{-3}\right)$ for the CCB preservative to the modified method of Boucherie.

\begin{tabular}{|c|c|c|c|}
\hline \multirow{3}{*}{$\begin{array}{l}\text { Fontes de } \\
\text { Variação }\end{array}$} & \multirow{3}{*}{$\begin{array}{c}\text { Graus de } \\
\text { Liberdade }\end{array}$} & \multicolumn{2}{|c|}{ Quadrado Médio } \\
\hline & & \multicolumn{2}{|c|}{ Boucherie Modificado } \\
\hline & & D. giganteus & B. vulgaris \\
\hline Concentração & 1 & $1,228^{*}$ & $1,689^{\mathrm{NS}}$ \\
\hline Resíduo & 2 & 0,035 & 1,505 \\
\hline
\end{tabular}

* significativo a $5 \%$ de probabilidade; ${ }^{\text {Ns }}$ não significativo a $5 \%$ de probabilidade.

Tabela 11. Retenções médias $\left(\mathrm{kg}\right.$ i.a.m $\left.\mathrm{m}^{-3}\right)$ para concentração nas peças, para a espécie $D$. giganteus.

Table 11. Retentions averages $\left(\mathrm{kg} \mathrm{a} \mathrm{i} . \mathrm{m}^{-3}\right)$ for concentration in stems for the species D. giganteus.

\begin{tabular}{cc} 
& Boucherie Modificado \\
\cline { 2 - 2 } Concentração (\%) & Dendrocalamus giganteus \\
\cline { 2 - 2 } & Retenção $\left(\mathbf{k g}\right.$ i.a.m. $\left.\mathbf{m}^{-3}\right)$ \\
\hline 3 & $0,28 \mathrm{~b}$ \\
\hline
\end{tabular}

As médias seguidas da mesma letra não diferem estatisticamente pelo teste de Tukey $(\mathrm{p} \geq 0,05)$.
A retenção de 2,2 kg.m ${ }^{-3}$ foi observada por Espelho (2007) em concentração de 5\%, em base sais, em tempo de tratamento de $90 \mathrm{~min}$, no tratamento de $D$. giganteus com idade superior a 5 anos. Pereira (1997) encontrou valores de 5,27 e 6,75 kg.m $\mathrm{m}^{-3}$ em colmos tratados em períodos de 4 e 15 horas, respectivamente, em 6\%, também em base sais; observe-se que, em ambos os trabalhos, empregou-se o método de Boucherie modificado.

\section{CONCLUSÕES}

Foi verificado que o valor da penetração de cobre e boro nos colmos das duas espécies de bambu foi baixo, sendo caracterizado entre penetração vascular e penetração parcial irregular. Entre as três regiões analisadas, foi verificado que houve maior penetração nos colmos na posição da base (0,5 m de altura).

A combinação de 15 dias de tratamento com 3\% de ingredientes ativos (i.a.) de CCB proporcionou melhor média de penetração da solução para os dois métodos de tratamento empregados.

As retenções dos i.a. do CCB obtidas nos dois métodos foram abaixo do recomendado pelas normas brasileiras para o tratamento de madeira. Foi observado, nas duas espécies, que as amostras tratadas com tempo de 15 dias e concentração de $3 \%$ de i.a. obtiveram valores mais elevados de retenção. Porém, é necessário realizar algumas modificações nas metodologias dos tratamentos preservativos, para que seja alcançada uma penetração e retenção mínima, recomendada pelas normas para tratamento de madeiras.

\section{STATUS DA SUBMISSÃO}

Recebido: 26 fev., 2013

Aceito: 14 ago., 2014

\section{AUTOR(ES) PARA CORRESPONDÊNCIA}

\section{Juarez Benigno Paes}

Departamento de Ciências Florestais e da Madeira, Universidade Federal do Espírito Santo - UFES, CEP 29550-000, Jerônimo Monteiro, ES, Brasil e-mail: jbp2@uol.com.br 


\section{REFERENNCIAS}

Adrianus R, Tambunan W, Supriyatin LK, Watimena C, Sudrajat $\mathrm{H}$, Yusuf M. Durability assessment of chemically treated Bambus ablumeana. World Journal of Fungal and Plant Biology 2010; 1(2): 32-36.

Associação Brasileira de Normas Técnicas - ABNT. NBR 6232: penetração e retenção de preservativos em madeira tratada sob pressão. Rio de Janeiro; 2013.

Associação Brasileira de Normas Técnicas - ABNT. NBR 9480: peças roliças preservadas de eucalipto para construções rurais: requisitos. Rio de Janeiro; 2009.

Azzini A, Beraldo AL. Métodos práticos para utilização do bambu. Campinas: UNICAMP; 2001.

Espelho JCC. Tratamento químico de colmos de bambu pelo método de Boucherie modificado [dissertação]. Campinas: Universidade Estadual de Campinas; 2007.

Ferreira LGR. Qualidade do tratamento preservativo do bambu [monografia]. Jerônimo Monteiro: Departamento de Engenharia Florestal, Universidade Federal do Espírito Santo; 2010.

Janssen JJA. Designing and building with bamboo. 20th ed. Netherlands: International Network for Bamboo and Rattan; 2000.

Liese W. Bamboos: biology, silvics, properties, utilization. Eschborn: Gesellschaft für Technische Zusammenarbeit; 1985.

Liese W. The anatomy of bamboo culms. 18th ed. Beijing: International Network for Bamboo and Rattan; 1998.

Paes JB, Moreschi JC, Lelles JG. Avaliação do tratamento preservativo de moirões de Eucalyptus viminalis Lab. e de bracatinga (Mimosa scabrella Benth.) pelo método de substituição da seiva. Ciência Florestal 2005; 15(1): 75-86.

Pereira MAR. O uso do bambu na irrigação - montagem de um sistema de irrigação por aspersão de pequeno porte, utilizando tubulação de bambu. In: Congresso Brasileiro de Engenharia Agrícola [CD-ROM]; 1997; Campina Grande. Campina Grande: CONBEA; 1997.

Pereira MAR, Beraldo AL. Bambu de corpo e alma. Bauru: Canal 6; 2008.

Rosa CAM, Santini EJ, Melo RR, Haselein CR, Stangerlin DM. Eficiência do tratamento preservativo de três espécies de bambu com CCB. In: Congresso Nordestino de Engenharia Florestal [CD-ROM]; 2009; Campina Grande. Campina Grande: UFCG; 2009.

Sales-Campos C, Vianez BF, Mendonça MS. Estudo da variabilidade da retenção do preservante CCA tipo A na madeira de Brosimum rubescens Taub. Moraceae - (paurainha) uma espécie madeireira da Região Amazônica. Revista Árvore 2003; 27(6): 845-853. http://dx.doi. org/10.1590/S0100-67622003000600011.

Santos RL, Lopes JDS. Construções com bambu: opção de baixo custo. Viçosa: Centro de Produções Técnica; 1998. Série Construções Rurais n. 160.

Torres PMA, Paes JB, Lira Filho JA, Nascimento JWB. Tratamento preservativo da madeira juvenil de Eucalyptus camaldulensis Dehnh. pelo método de substituição de seiva. Cerne 2011; 17(2): 275-282. http://dx.doi.org/10.1590/ S0104-77602011000200016.

Ubidia JAM. Preservación del bambú en America Latina, mediante métodos tradicionales [cited 2012 May 19]. Available from: http://www.inbar.int/publications/?did=131. 